\title{
Luminance Prediction of Paper Model Surface Based on Non-Contact Measurement
}

\author{
Xiaozhou LI*, Xiaomeng HAN, Jingjing LIU, Xuelin LI, Mingming CUI, Guangyuan WU
}

\begin{abstract}
The overall appearance perception is affected by luminance perception accuracy and efficiency mostly. The surface luminance prediction correlated with surface angle and surface tone value was performed by measuring and modeling the paper model surface luminance. First, we used a rotating bracket designed to facilitate to set the paper surface angle. Then, we set the surface angle from $5^{\circ}$ to $85^{\circ}$ at the interval of $5^{\circ}$ using the designed rotating bracket. Additionally, the four primary color scales, cyan, magenta, yellow, and black, were printed and set at the designed angle. The angle-ware and tone-ware luminance was measured using spectroradiometer, CS-2000. Finally, we proposed and evaluated a mathematical model to reveal the relationship between luminance and surface angle and surface tone using the least squares method. The results indicated that the surface luminance of paper model could be predicted and obtained quickly and accurately for any surface angles and surface tone values by the proposed prediction model.
\end{abstract}

Keywords: luminance prediction model; non-contact measurement; paper model surface; surface angle; surface luminance prediction; surface tone

\section{INTRODUCTION}

Luminance prediction and analysis of object surfaces is becoming a hot research topic in the area related to colour reproduction, stereoscopic image processing and 3D printing. The luminance perception is made up of the total appearance perception for the physical objects. Luminance perception can also make more effects on the appearance perception and the details representation is also determined by the luminance perception, which contributes to object surface colour perception [1]. However, there are giant differences between the viewing conditions for 2D plain image and 3D stereoscopic image [2]. For 2D plain image perception, luminance perception, measurement, or reproduction is a mature technique used in the area of many research and industrial areas, such as printing colour reproduction [3]. Luminance perception is always restricted to deposit the measure point or focus at a given angle on the surface. Luminance can be measured easily with contact measurement or prediction models [4]. Similarly to luminance perception on 2D plain surface, the given point can be measured using contact measure method and instrument. In contrast, due to the irregular geometric shape of the 3D object surface, the luminance always gives different perception for the same viewing point. It is difficult to get total luminance for the 3D object surface with irregular geometric shape due to the limits of the measuring instruments, prediction models and complexity of viewing conditions [5]. Therefore, it is still necessary to do further research on the luminance perception and prediction of the $3 \mathrm{D}$ object surface with a simple method in colour research areas [6]. On one hand, it is easy to get the total appearance of 3D object with the help of 3D scanner. But the information obtained from the $3 \mathrm{D}$ object surface is almost about the distance information which is used to create the surface point cloud information to construct the object surface shape. It does not include the surface luminance and colour information which is obviously different from the information obtained using 2D scanner that mostly includes luminance information and colour information [7]. On the other hand, perception, prediction and representation of 3D object surface colour information must work with the help of luminance. It is not enough to perform the colour representation only with 3D scanner [8]. How to predict the luminance of 3D object surface is becoming more and more important in such area. In this work, paper model was used to work as the primary object to represent luminance perception and prediction with the basic surface geometric and tone information. Surface angle and tone value are the main properties in such paper model surface geometric information. The function happening between light and paper on 3D paper model surface is similar to that on $2 \mathrm{D}$ plain paper surface from the perspective of micro-structure which is always described by the BRDF theory [9]. The paper surface angle and tone value relative to the observer were set to be noncontact measured luminance using spectroradiometer in our work. The relations between luminance and bi-factors, surface angle and tone value, were analysed to get the luminance perception and prediction of paper model surface. Paper surface luminance is affected by the interaction between paper and inks, paper surface roughness and other parameters [10]. Just like the human skin colour and luminance measurement, skin surface is processed specially to obtain the colour perception under different viewing angles [11], while the paper surface is processed to measure the luminance using four primary colour inks.

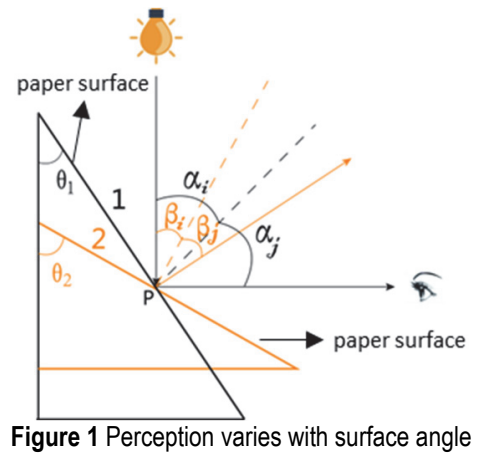

Paper model surface is set in the exact viewing conditions, i.e., the lighting source position and observer viewing angles are fixed. Obviously, there are various surface angles against the observer for the irregular geometric surface of paper model. The perception caused by surface angle can be shown in Fig. 1. For paper model surfaces, its angles against vertical line or horizontal line 
are various. For surface 1 , the vertical angle is $\theta_{1}$, and the incident angle to the point $P$ is $\alpha_{i}$ and the reflection angle is $\alpha_{j}$. However, to the surface 2 whose angle is $\theta_{2}$, its incident angle and reflection angle will change to $\beta_{i}$ and $\beta_{j}$, though the model position, light source position and perceiver position keep the same as before. It will cause the lightness, i.e., luminance, to change obviously.

Microscopically speaking, the surface of paper model is similar to that of $2 \mathrm{D}$ plain paper, it has the same interaction between light and paper surface which is always described by BRDF and related theories [13]. To be noted that interaction occurring on the microfacet with light is shadowing and masking. Shadowing is the occlusion of the light source by the microgeometry while masking is the visibility occlusion of a microfacet by another microgeometry (see Fig. 2).

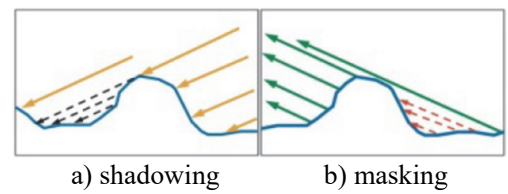

Figure 2 Shadowing and Masking on the microfacet

Actually, there is always interreflection between the microfacets or other optical interactions between the surface and the paper inside, such as scattering, absorption and refraction (see Fig. 3). Anyway, these optical matters are always ignored in measuring the surface lightness. We would not take that factor into account in our paper and it is consistent with the practical prediction and measurement.

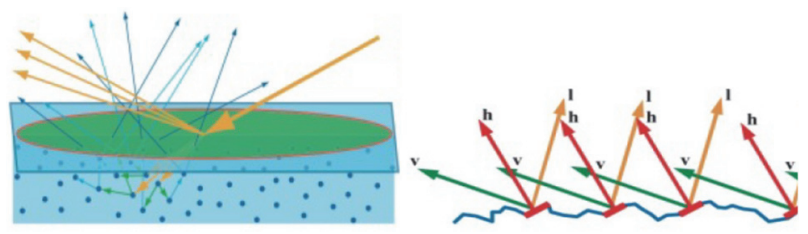

Figure 3 Interreflection between the micorfacets on the surface

\section{EXPERIMENT}

\subsection{Paper Model Surface Sample Design}

We use electrophotography press, Konica Minolta (KM-C6000), to output colour patches with paper of 128 $\mathrm{g} / \mathrm{m}^{2}$. KM-C6000 is the colour equipment that uses electrophotography technique to output the colours on the paper surface. Its toner is controlled by electrostatic force and forms images on paper. The ink set we used is TN616 which includes Cyan, Magenta, Yellow and Black. TN616 is a group of Toner Cartridge for Konica Minolta.

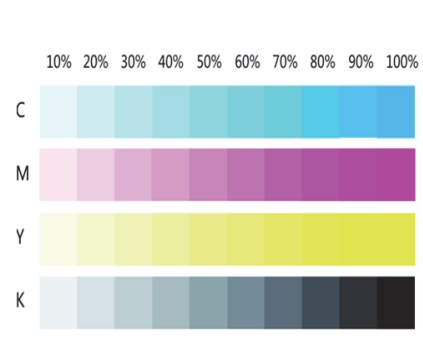

a) CMYKcolor chart

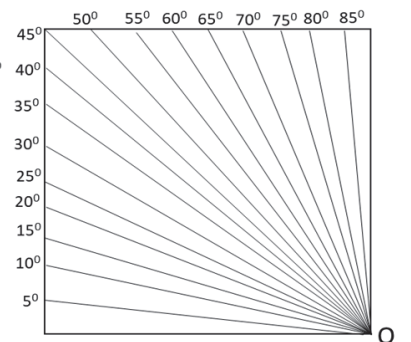

b) paper model surface angle set
Figure 4 Tone scale and angle set of paper model surface
Tone chart, $S$, was designed from $10 \%$ to $100 \%$ with intervals of $10 \%$. Here $S$ is a group primary colour chart. The size of each colour patch is $3 \mathrm{~cm} \times 3 \mathrm{~cm}$, Fig. $4 \mathrm{a}$. Model sample surface angles were designed from $5^{\circ}$ to $85^{\circ}$ with interval of $5^{\circ}$, Fig. $4 \mathrm{~b}$.

\subsection{Measuring Conditions Set}

We choose the non-contact method to measure the luminance for the paper model surface, and spectroradiometer, CS2000, was used to obtain the data from the paper sample surface. CS2000 is a high accuracy spectroradiometer designed to measure luminance and chromaticity up to super-low luminance regions. Paper samples were set in a standard light source box with D65.

The light box used in the experiment is suitable for the need to keep colour consistency and quality. It has multipoint source observation system, used in printing ink, paint, plastics, cosmetics, textiles, leather, food, paper, stain and other non-ferrous materials inspection and match colours. The colour box is made of solid material of medium density fiberboard, lighting box inside is TuMeng N5.5 self gray. And such light box is equipped with membrane switch, coming with a 45 degree tilt observatory and five standard light sources. They are F/A, TL84, D65, D50, UV.

All measurement data was obtained in darkroom which excludes the effect of environment light. The bracket used in this experiment was to help set the paper sample surface angle effectively and accurately [12]. The point measured should be kept the same for every surface and the measure distance should also be kept the same for every sample. Additionally, the vertical distance from the light to the sample surface should be kept the same for different samples. The experiment condition set is shown in Fig. 5. According to our experimental conditions, measuring distance is set as $172 \mathrm{~cm}$. and the vertical distance was set as $49 \mathrm{~cm}$.

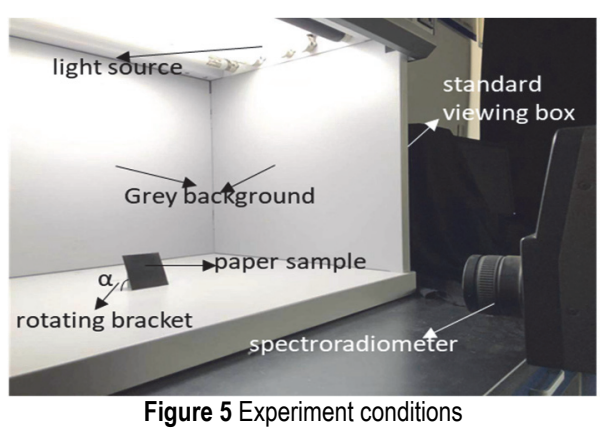

Table 1 Effect of measurement distances

Table 1 Effect of measurement distances
\begin{tabular}{|c|c|c|c|c|c|}
\hline Horizonta1/cm & $\mathrm{X}(1931)$ & $\mathrm{Y}(1931)$ & $\mathrm{Z}(1931)$ & $\mathrm{x}(1931)$ & $\mathrm{y}(1931)$ \\
\hline 100 & 50.78 & 48.52 & 55.98 & 0.3270 & 0.3125 \\
\hline 150 & 51.42 & 49.13 & 56.68 & 0.3270 & 0.3125 \\
\hline 172 & 52.16 & 49.84 & 57.54 & 0.3269 & 0.3124 \\
\hline 200 & 51.46 & 49.16 & 56.78 & 0.3269 & 0.3123 \\
\hline
\end{tabular}

The chromatic coordinate difference is too small to perceive for different horizontal and vertical measuring differences (see Tab. 1 and Tab. 2). It showed that there is less effect from the horizontal and vertical measure distance in the experiment viewing conditions. 
Table 2 Effect of vertical distances to light source

\begin{tabular}{|c|c|c|c|c|c|}
\hline Vertical / cm & $\mathrm{X}(1931)$ & $\mathrm{Y}(1931)$ & $\mathrm{Z}(1931)$ & $\mathrm{x}(1931)$ & $\mathrm{y}(1931)$ \\
\hline 49.5 & 52.72 & 50.37 & 58.07 & 0.3271 & 0.3125 \\
\hline 49 & 54.25 & 51.84 & 59.80 & 0.3270 & 0.3125 \\
\hline 48 & 53.87 & 51.27 & 60.08 & 0.3261 & 0.3103 \\
\hline 47 & 57.15 & 55.16 & 61.98 & 0.3279 & 0.3165 \\
\hline
\end{tabular}

\section{RESULT AND DISCUSSION}

\subsection{Effects of Surface Angle on Luminance}

The paper model surface luminance of angle-aware and tone-aware was measured. And the luminance values, surface angles and surface tone values were normalized to 0 to 1 . The relations between the luminance and surface angle with different tone values are shown in Fig. 6. Obviously, there is a mutation point at the angle of $30^{\circ}$ for every tone of $\mathrm{C}, \mathrm{M}, \mathrm{Y}$ and $\mathrm{K}$. Furthermore, $\mathrm{Y}$ has another mutation point at $60^{\circ}$, though it is less obvious compared with that of $30^{\circ}$. At $30^{\circ}$, luminance becomes higher for every sample, which is consistent with the objective perception while the effect of $30^{\circ}$ for each tone value on luminance is smaller for $\mathrm{Y}$ primary tone compared with the other three primary colour tones. In Fig. $4, L v$ is luminance and angle is surface angle.

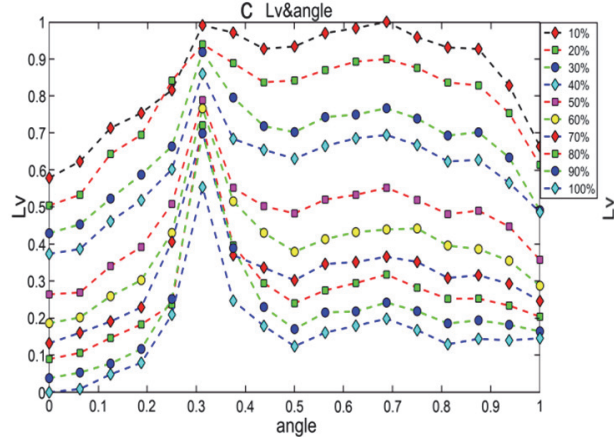

a) luminance vs surface angle for Cyan chart

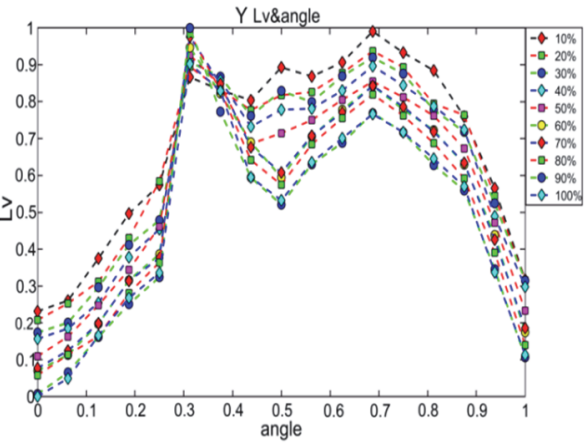

c) luminance vs surface angle for Yellow chart

Figure 6 Effect of surface angle on luminance for four primary tones

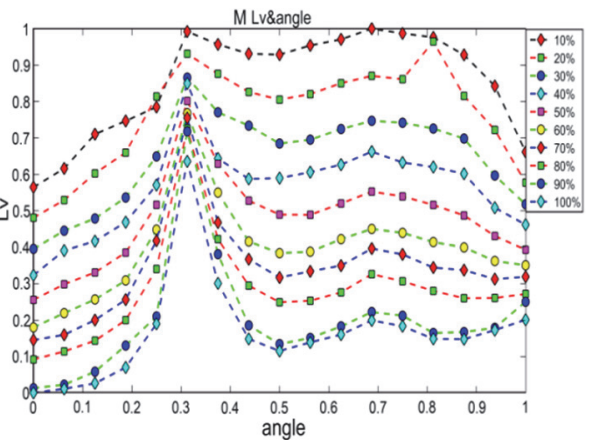

b) luminance vs surface angle for Magenta chart

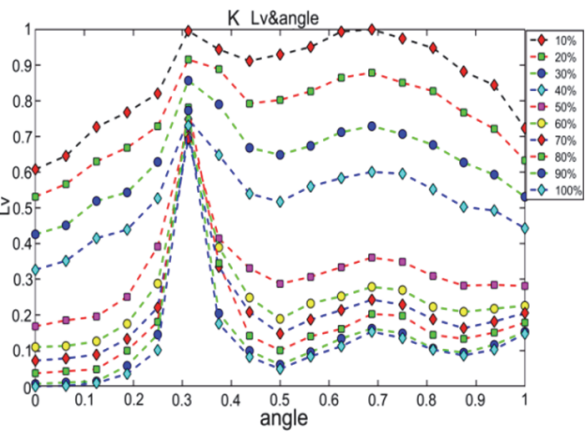

d) luminance vs surface angle for Black chart

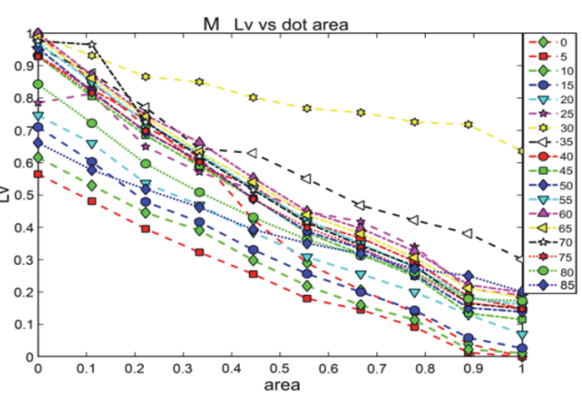

b) luminance vs surface tone for Magenta chart

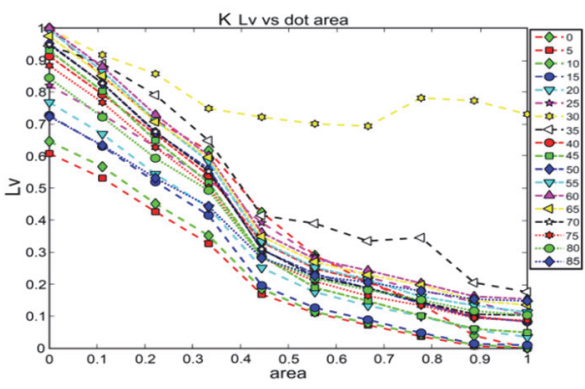

d) luminance vs surface tone for Black chart

c) luminance vs surface tone for Yellow chart

Figure 7 Effect of primary colour tones on luminance for various surface angles 


\subsection{Effects of Tone Value on Luminance}

Luminance decreases with the increase of tone values in $2 \mathrm{D}$ printing process. For 3D paper model surface, luminance perception is affected by the tone value and surface angle simultaneously (see Fig. 5). The luminance of $30^{\circ}$ surface has the brightest perception for all samples. It shows that the luminance perceived on $30^{\circ}$ surface is less affected no matter the primary colour tone changes. For the same surface angle, luminance has similar trends to those that happened in $2 \mathrm{D}$ printing. Obviously, the $\mathrm{Y}$ primary tone has more dispersed luminance perceptions than other three primary tones. In Fig. 5, $L v$ is luminance and dot area is surface tone value. And the luminance values, surface angles and surface tone values were normalized to 0 to 1 .

\section{LUMINANCE PREDICTION MODELLING AND ANALYSIS}

\subsection{Modelling}

There are several sets of equations between the luminance and tone values, luminance and surface angles that have not been determined. The least squares method [14] is used to approximate the solution of the surface angle and tone value on the luminance perception for paper model surface. From Fig. 4 and Fig. 5, luminance is affected by tone value obviously and the relation can be shown as follows:

$L v_{i}=a_{i} \times S_{i}+b_{i}, i=1,2, \ldots, n$

where, $L v_{i}$ is luminance, $S_{i}$ is tone value, $a_{i}$ and $b_{i}$ are scale factor, $i$ is the ordinal number of surface angle. For different surface angles, we rewrite the Eq. (1) as follows:

$$
\left[\begin{array}{c}
L v_{1} \\
L v_{2} \\
\ldots \\
L v_{n}
\end{array}\right]=\left[\begin{array}{ccc}
S_{11} & \ldots & S_{1 n} \\
\ldots & \ldots & \ldots \\
S_{n 1} & \ldots & S_{n n}
\end{array}\right]\left[\begin{array}{c}
a_{1} \\
a_{2} \\
\ldots \\
a_{n}
\end{array}\right]
$$

With $\left[\begin{array}{c}L v_{1} \\ L v_{2} \\ \ldots \\ L v_{n}\end{array}\right]=Y,\left[\begin{array}{ccc}S_{11} & \ldots & S_{1 n} \\ \ldots & \ldots & \ldots \\ S_{n 1} & \ldots & S_{n n}\end{array}\right]=C,\left[\begin{array}{c}a_{1} \\ a_{2} \\ \ldots \\ a_{n}\end{array}\right]=X \quad Y$ and $C$ are the samples, $X$ is scale factor. The Eq. (2) can be rewritten as follows:

$$
C X=Y
$$

In Eq. (3), we can get:

$$
\boldsymbol{C}^{T} \boldsymbol{C} X=\boldsymbol{C}^{T} Y
$$

where, $\boldsymbol{C}^{T}$ is transpose matrix of $\boldsymbol{C}$, so we can rewrite Eq. (4) as follows:

$$
X=\left(\boldsymbol{C}^{T} \boldsymbol{C}\right)^{-1} \boldsymbol{C}^{T} Y
$$

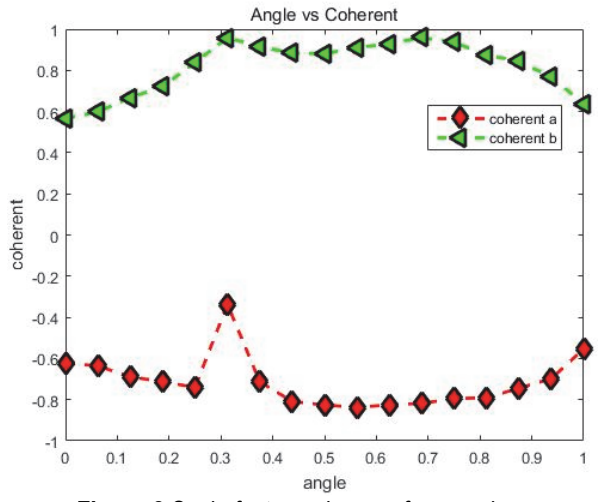

Figure 8 Scale factor $a, b$ vs surface angle $a$

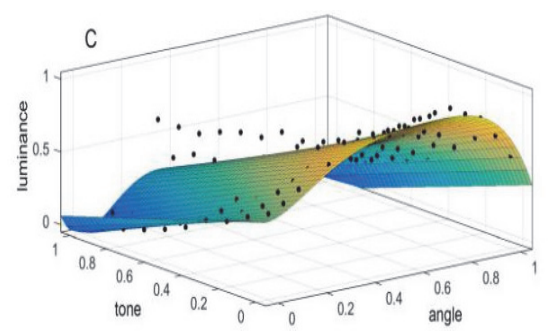

a) luminance vs surface angle \& surface tone for Cyan

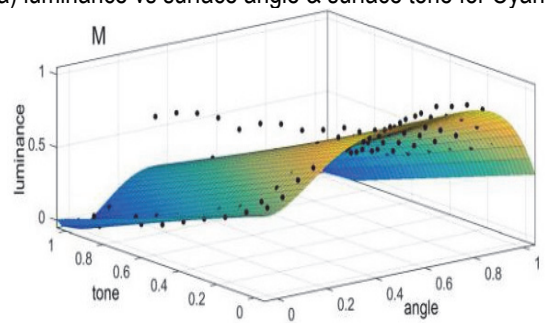

b) luminance vs surface angle \& surface tone for Magenta

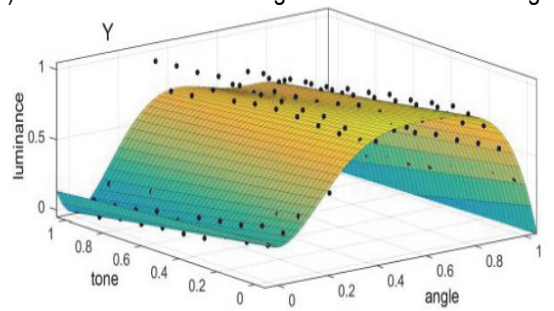

c) luminance vs surface angle \& surface tone for Yellow

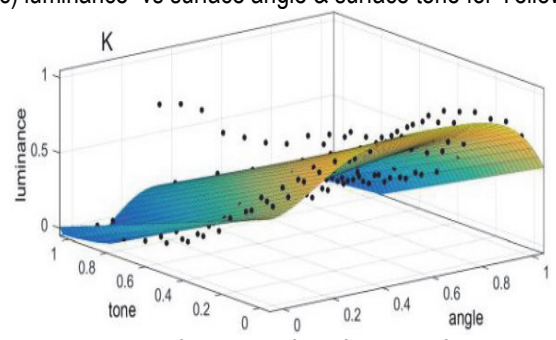

d) luminance vs surface angle \& surface tone for Black Figure 9 Luminance Predicted vs Luminance Measured: Colored continuous belts: Luminance predicted; Black points: Luminance measured

We need to solve this equation to calculate the scale factors of every surface angle $\alpha$. For our 17 designed surface angles, one set of scale factors $a_{i}$ and $b_{i}$ were obtained as in Fig. 6. It showed that $a$ and $b$ can be calculated as functions of surface angle $\alpha$ as follows:

$\left\{\begin{array}{l}a=g(\alpha) \\ b=t(\alpha)\end{array}\right.$ 
where, we chose fifth-order nonlinear function to calculate the relation between scale factor and surface angle and finally converted the intrinsic relations between luminance and tone to intrinsic relations between luminance and tone $\&$ surface angle, as follows:

$L v=F(S, \alpha)=g(\alpha) \times S+t(\alpha)$

We can compute the luminance prediction for four primary colour tone samples according to our proposed model, Eq. (5) and Eq. (7). The results are shown in Fig. 7. Black points are the sample luminance measured using our experimental sets. The colour surfaces are the predicted luminance using the model proposed in this paper. It showed that most of the points matched the model well except the $30^{\circ}$. We will further discuss the performance of our proposed prediction model when put in practice in the next section.

\subsection{Analysis}

We use $R^{2}$ (coefficient of determination) to analyze the proposed model. $R^{2}$ is the proportion of variance in the dependent variable from the independent variables [15]. It is a static that gives information about the goodness of fit of our model. $R^{2}$ can be defined as follows [13]:

$$
R^{2}=1-\frac{\sum\left(y_{i}-\hat{y}_{\mathrm{i}}\right)^{2}}{\sum\left(y_{i}-\bar{y}_{i}\right)^{2}}
$$

where, $y_{i}$ is sample value, $\hat{y_{i}}$ is predicted value, $\overline{\mathrm{y}_{i}}$ is average value of samples, $n$ is the total number of samples. Values of $R^{2}$ are from 0 to 1 . An $R^{2}$ of 0 indicates that the fitting prediction values does not match the measurement values at all while a $R^{2}$ of 1 indicates that the prediction values perfectly fit the data. In this experiment, $R^{2}$ of four primary color samples were computed as in Tab. 3. Both values of $R^{2}$ of Cyan and Magenta are over 0.9 which shows the fitting process matches well with the measurement, i.e., over $90 \%$ of fitting prediction luminance data matched the measured data. And values of $R^{2}$ of Yellow and Black are over 0.8 which shows the fitting process is still accepted and over $80 \%$ of fitting prediction luminance data matched the measured data.

Table 3 Values of $R^{2}$ of four primary color samples

\begin{tabular}{|c|c|c|c|c|}
\hline & $\mathrm{C}$ & $\mathrm{M}$ & $\mathrm{Y}$ & $\mathrm{K}$ \\
\hline$R^{2}$ & 0.9115 & 0.9013 & 0.8747 & 0.8268 \\
\hline
\end{tabular}

Additionally, according to the model proposed in Eq. (5) and Eq. (7), all samples of designed surface angles with primary color tones were computed and measured. The results are shown in Tab. 4 . It shows that the difference $\Delta$ between prediction value and measured value is from 0.01 to 8.06. The average difference of yellow is the smallest while the black is the biggest. The average value of the total differences is 3.03. And the minimum differences of all primary color samples almost happened at $30^{\circ}$ surface angle or its neighbor point.

Table 4 Prediction values vs Measured values for designed surface angles

\begin{tabular}{|c|c|c|c|c|c|c|c|c|c|c|c|c|c|}
\hline \multirow{2}{*}{$\begin{array}{c}\text { Surface- } \\
\text { Angle }\end{array}$} & \multirow{2}{*}{$\begin{array}{c}\text { Unprinted } \\
\text { Paper } \\
\text { Surface }\end{array}$} & \multicolumn{3}{|c|}{ C-tone } & \multicolumn{3}{|c|}{ M-tone } & \multicolumn{3}{|c|}{ Y-tone } & \multicolumn{3}{|c|}{ K-tone } \\
\hline & & Mea. & Pre. & $\Delta$ & Mea. & Pre. & $\Delta$ & Mea. & Pre. & $\Delta$ & Mea. & Pre. & $\Delta$ \\
\hline 5 & 43.018 & 50.56 & 48.69 & 1.87 & 53.40 & 51.18 & 2.22 & 61.04 & 61.13 & -0.09 & 51.21 & 46.81 & 4.40 \\
\hline 10 & 44.473 & 53.40 & 51.51 & 1.89 & 56.30 & 54.08 & 2.22 & 64.21 & 64.20 & 0.01 & 54.90 & 50.11 & 4.79 \\
\hline 15 & 44.154 & 59.18 & 57.33 & 1.85 & 61.39 & 59.20 & 2.19 & 68.96 & 68.74 & 0.22 & 61.86 & 56.92 & 4.95 \\
\hline 20 & 48.55 & 61.77 & 60.05 & 1.71 & 65.33 & 63.33 & 2.00 & 73.68 & 73.50 & 0.19 & 64.72 & 60.29 & 4.43 \\
\hline 25 & 48.484 & 65.73 & 65.81 & -0.08 & 72.05 & 70.25 & 1.80 & 74.94 & 76.28 & -1.34 & 70.92 & 66.72 & 4.21 \\
\hline 30 & 50.977 & 76.92 & 75.80 & 1.21 & 87.04 & 85.92 & 1.21 & 96.39 & 99.40 & -3.02 & 84.69 & 83.08 & 1.62 \\
\hline 35 & 56.265 & 75.65 & 73.59 & 2.06 & 82.99 & 80.79 & 2.19 & 90.33 & 91.97 & -1.64 & 81.57 & 77.06 & 4.51 \\
\hline 40 & 59.63 & 72.86 & 70.44 & 2.42 & 81.58 & 78.52 & 3.07 & 89.68 & 89.67 & 0.01 & 79.11 & 73.59 & 5.55 \\
\hline 45 & 62.73 & 73.26 & 70.30 & 2.97 & 81.85 & 78.13 & 3.72 & 93.99 & 93.85 & 0.14 & 81.60 & 74.82 & 6.78 \\
\hline 50 & 64.665 & 75.56 & 72.30 & 3.26 & 83.70 & 79.70 & 4.00 & 92.51 & 92.29 & 0.22 & 82.98 & 75.63 & 7.36 \\
\hline 55 & 67.827 & 76.44 & 72.99 & 3.44 & 84.58 & 80.42 & 4.15 & 94.19 & 93.83 & 0.36 & 84.69 & 76.80 & 7.88 \\
\hline 60 & 71.594 & 77.50 & 73.80 & 3.50 & 86.83 & 82.59 & 4.24 & 97.36 & 96.89 & 0.47 & 85.78 & 77.72 & 8.06 \\
\hline 65 & 74.386 & 74.87 & 71.45 & 3.42 & 83.71 & 79.46 & 4.25 & 96.75 & 96.14 & 0.61 & 83.68 & 75.70 & 7.98 \\
\hline 70 & 76.609 & 73.10 & 69.72 & 3.38 & 86.52 & 82.29 & 4.23 & 92.78 & 92.18 & 0.60 & 80.73 & 72.95 & 7.78 \\
\hline 75 & 82.324 & 72.83 & 69.52 & 3.31 & 83.36 & 79.18 & 4.18 & 87.58 & 87.25 & 0.33 & 75.91 & 68.68 & 7.22 \\
\hline 80 & 85.318 & 66.52 & 63.34 & 3.18 & 72.51 & 68.71 & 3.79 & 78.64 & 78.82 & -0.18 & 71.12 & 64.45 & 6.67 \\
\hline 85 & 87.793 & 55.99 & 52.99 & 3.00 & 61.85 & 58.60 & 3.25 & 67.04 & 68.25 & -1.21 & 60.48 & 54.70 & 5.78 \\
\hline Ave. $\Delta$ & -- & \multicolumn{3}{|c|}{2.50} & \multicolumn{3}{|c|}{3.10} & \multicolumn{3}{|c|}{0.63} & \multicolumn{3}{|c|}{5.88} \\
\hline
\end{tabular}

Additionally, we chose 7 arbitrary surface angles and used the proposed model to get the prediction luminance value of paper model surface. The surface color tones are still from $10 \%$ to $100 \%$. It is shown in Tab. 5. The highest value of luminance differences is 7.96 and the lowest value of luminance differences is 0.11 . The average value of luminance differences is 2.62. Most of the luminance differences can almost not be perceived by human eyes. The results indicate that luminance of arbitrary surface angle and tone values can be predicted using our proposed model. 
Tab. 5 Prediction values vs Measured values for arbitrary surface angles

\begin{tabular}{|c|c|c|c|c|c|c|c|c|c|c|c|c|}
\hline \multirow{2}{*}{$\begin{array}{l}\text { Surface- } \\
\text { Angle }\end{array}$} & \multicolumn{3}{|c|}{ C-Tone } & \multicolumn{3}{|c|}{ M-Tone } & \multicolumn{3}{|c|}{ Y-Tone } & \multicolumn{3}{|c|}{ K-Tone } \\
\hline & Mea. & Pre. & $\Delta$ & Mea. & Pre. & $\Delta$ & Mea. & Pre. & $\Delta$ & Mea. & Pre. & $\Delta$ \\
\hline 17 & 62.31 & 59.48 & 2.83 & 59.27 & 57.47 & 1.80 & 66.15 & 66.26 & -0.11 & 58.36 & 53.43 & 4.93 \\
\hline 28 & 78.45 & 75.19 & 3.26 & 74.59 & 72.57 & 2.03 & 82.57 & 90.47 & -7.9 & 74.48 & 70.01 & 4.47 \\
\hline 33 & 83.27 & 80.27 & 3.00 & 82.20 & 81.48 & 0.72 & 91.47 & 98.29 & -6.82 & 82.28 & 80.81 & 1.47 \\
\hline 42 & 81.16 & 77.16 & 4.00 & 76.81 & 75.38 & 1.43 & 84.92 & 83.02 & 1.9 & 75.10 & 70.75 & 4.45 \\
\hline 48 & 80.53 & 77.74 & 2.79 & 79.16 & 76.85 & 2.31 & 85.63 & 85.11 & 0.52 & 76.38 & 71.47 & 4.91 \\
\hline 57 & 86.79 & 82.73 & 4.06 & 83.10 & 80.18 & 2.92 & 95.61 & 95.30 & 0.31 & 81.39 & 74.41 & 6.98 \\
\hline 62 & 87.62 & 82.62 & 5.00 & 83.14 & 80.56 & 2.58 & 93.63 & 93.41 & 0.22 & 81.26 & 73.30 & 7.96 \\
\hline Ave. $\Delta$ & \multicolumn{3}{|c|}{3.56} & \multicolumn{3}{|c|}{1.97} & \multicolumn{3}{|c|}{2.54} & \multicolumn{3}{|c|}{5.02} \\
\hline
\end{tabular}

\section{CONCLUSION}

The surface luminance prediction based on different surface angles and surface tone values was performed by measuring and modeling the paper model surface luminance. We set the angle from $5^{\circ}$ to $85^{\circ}$ at the interval of $5^{\circ}$. In addition, four primary color scales, cyan, magenta, yellow, and black, were printed and set at paper model surface. The angle-ware and tone-ware luminance was measured using non-contact measuring method. Finally, we proposed and evaluated a mathematical model to reveal the relationship between luminance and surface angles and surface tones. It indicated that the surface luminance of paper model could be predicted and obtained quickly and accurately by the proposed prediction model.

\section{Acknowledgement}

This work was financially supported by Key Research and Development Program of Shandong Province (2019GGX105016, 2018GGX106009), State Scholarship Fund of China (201808370100), Scientific Research Foundation of Higher Education Institutions of Shandong Province (J18KA332, J17KA178), National Undergraduate Training Program on Innovation and Entrepreneurship (201810431003), Foundation of Key Laboratory of Pulp and Paper Science and Technology of Ministry of Education/Shandong Province of China(KF201604), State Key Laboratory of Pulp and Paper Engineering(201722), Foundation of Shandong University of Art \& Design(X19KY31).

\section{REFERENCES}

[1] Dana, K. J., Ginneken, B. V., Nayar, S. K., \& Koenderink, J. J. (1999). Reflectance and texture of real-world surfaces. ACM Transactions on Graphics, 18(1), 1-34. https://doi.org/10.1145/300776.300778

[2] Sumin, D., Weyrich, T., Rittig, T., Babaei, V., Nindel, T., Wilkie, A., \& Myszkowski, K. (2019). Geometry-aware scattering compensation for 3D printing. ACM Transactions on Graphics, 38(4), 1-14. https://doi.org/10.1145/3306346.3322992

[3] Babaei, V., Vidimče, K., Foshey, M., Kaspar, A., Didyk, P., \& Matusik, W. (2017). Color contoning for 3D printing. ACM Transactions on Graphics, 36(4), 1-15. https://doi.org/10.1145/3072959.3073605

[4] Shevell, S. K. (2008).The Science of Color $2^{\text {nd }}$. Amsterdam: Elsevier.

[5] Xiaozhou, L. (2012). Study on Color Gamut Mapping Theory and Method Based on Color Appearance Model of High Fidelity Color Reproduction. Guangzhou: South China University of Technology.
[6] Lian, Z., Rosin, P. L., \& Sun, X. (2009). Rectilinearity of 3D Meshes. International Journal of Computer Vision, 89(2-3), 130-151. https://doi.org/10.1007/s11263-009-0295-0

[7] Kurban, T. \& Besdok, E. (2015). Design, Implementation and Comparison of Low-Cost Laser Scanning Systems for 3D Modeling. The 7th International Conference on Information Technology. https://doi.org/10.15849/icit.2015.0097

[8] Hess, M. (2017). 3D Laser Scanning. Digital Techniques for Documenting and Preserving Cultural Heritage. https://doi.org/10.5040/9781641899444.009

[9] Kijanka, P. \& Packo, P. (2019). Novel method for true guided waves spectral characteristics estimation using a logistic function fit and nonlinear least square algorithm. Structural Control and Health Monitoring, 26(9). https://doi.org/10.1002/stc.2388

[10] Pravdova, V., Walczak, B., \& Massart, D. (2002). A comparison of two algorithms for warping of analytical signals. Analytica Chimica Acta, 456(1), 77-92. https://doi.org/10.1016/S0003-2670(02)00008-9

[11] Ikeda, N., Miyashita, K., Hikima, R., \& Tominaga, S. (2014). Reflection measurement and visual evaluation of the luminosity of skin coated with powder foundation. Color Research \& Application, 39(1), 45-56. https://doi.org/10.1002/col.21753

[12] Li, X. Z., Sun, F. Y., Cui, M. M., et al. (2019). Angle measuring tool. China Patent: CN209147902U, 2019-07-23.

[13] Ikemoto, N., Fujinami, K., \& Isomura, M. (2002). Modeling the Luminance Distribution of 3-D Object Surface with Specular Reflection Component and Shape Measurement. Journal of Light \& Visual Environment, 26(2), 19-28. https://doi.org/10.2150/jlve.26.2_19

[14] Least squares data fitting. (2018). Introduction to Applied Linear Algebra, 245-284. https://doi.org/10.1017/9781108583664.014

[15] Neath, A. A., Flores, J. E., \& Cavanaugh, J. E. (2017). Bayesian multiple comparisons and model selection. Wiley Interdisciplinary Reviews: Computational Statistics, 10(2). https://doi.org/10.1002/wics.1420 


\section{Contact information:}

Xiaozhou LI, PhD. Associate Prof.

(Corresponding author)

School of Light Industry Science and Engineering, Qilu University of Technology

(Shandong Academy of Sciences)

3501 Daxue Road, Jinan, Shandong, China, 250300

Email: lixiaozhou2000@163.com

Xiaomeng HAN, Ms

School of Light Industry Science and Engineering, Qilu University of Technology

(Shandong Academy of Sciences)

3501 Daxue Road, Jinan, Shandong, China, 250300

E-mail: 2282839896@qq.com

Jingjing LIU, Associate Prof.

Shandong University of Art \& Design

1255 Daxue Road, Jinan, Shandong, China, 250300

Email: foxliu919@163.com

Xuelin LI, Associate Prof.

Qilu University of Technology (Shandong Academy of Sciences)

3501 Daxue Road, Jinan, Shandong, China, 250300

Email: lixiaozhou2000@163.com

\section{Mingming CUI, Ms.}

School of Light Industry Science and Engineering, Qilu University of Technology (Shandong Academy of Sciences)

3501 Daxue Road, Jinan, Shandong, China, 250300

E-mail: 751867202@qq.com

\section{Guangyuan WU, PhD}

School of Light Industry Science and Engineering, Qilu University of Technology (Shandong Academy of Sciences)

3501 Daxue Road, Jinan, Shandong, China, 250300

E-mail: wgy19882000@163.com 